OPEN

SUBJECT AREAS:

GALAXIES AND CLUSTERS

PALAEOCLIMATE

Received

31 March 2014

Accepted

1 August 2014

Published

21 August 2014

Correspondence and requests for materials should be addressed to N.J.S. (shaviv@phys. huji.ac.il)

\section{Is the Solar System's Galactic Motion Imprinted in the Phanerozoic Climate?}

\author{
Nir J. Shaviv' , Andreas Prokoph² \& Ján Veizer ${ }^{3}$
}

\author{
${ }^{1}$ Racah Institute of Physics, Hebrew University of Jerusalem, Jerusalem 91904, Israel, ${ }^{2}$ Speedstat, Ottawa ON K 1G 5J5, Canada, \\ ${ }^{3}$ Ottawa-Carleton Geoscience Center, University of Ottawa, Ottawa ON K1N 6N5, Canada.
}

A new $\delta^{18} \mathrm{O}$ Phanerozoic database, based on 24,000 low-Mg calcitic fossil shells, yields a prominent $32 \mathrm{Ma}$ oscillation with a secondary $175 \mathrm{Ma}$ frequency modulation. The periodicities and phases of these oscillations are consistent with parameters postulated for the vertical motion of the solar system across the galactic plane, modulated by the radial epicyclic motion. We propose therefore that the galactic motion left an imprint on the terrestrial climate record. Based on its vertical motion, the effective average galactic density encountered by the solar system is $0.172 \pm 0.006$ stat \pm 0.006 sys $\mathrm{M}_{\odot} \mathrm{pc}^{-3}$. This suggests the presence of a disk dark matter component.

rear he Milky Way is a barred spiral galaxy. As a consequence, the solar system revolves around the galaxy and carries out a small oscillation in the direction perpendicular to the dense galactic disk, with a period much shorter than its orbital period ${ }^{1}$. For very small amplitudes of oscillation, the disk-crossing period $P_{1 / 2}$ depends solely on the density $\rho_{0}$ in the galactic plane, via $P_{1 / 2}=\left(\pi / 4 G \rho_{0}\right)^{1 / 2}=13.2\left(\rho_{0} /\left[\mathrm{M}_{\odot} \mathrm{pc}^{-3}\right]\right)^{-1 / 2} \mathrm{Ma}$ (with $G$ being the gravitational constant, assuming a constant density disk, otherwise the period may be somewhat larger ${ }^{2}$ ). Previous determinations of the half-period range between 30 and $42 \mathrm{Ma}^{2-4}$. The methodologies for measuring the density of the galactic disk can be classified into three categories. In the first approach, the known components are simply summed up, giving the total baryonic mass density at the galactic plane of about 0.09 to $0.10 \mathrm{M}_{\odot} \mathrm{pc}^{-3} 4,5$. The second approach is based on the vertical kinematics of stars ${ }^{6}$. Assuming that a given tracer population of stars is kinematically relaxed, there is then a relation between the vertical density of the tracer stars and their dispersion velocity. This enables derivation of the underlying vertical dependence of the gravitational potential, and from it the density, yielding values from 0.07 to $0.26 \mathrm{M}_{\odot} \mathrm{pc}^{-32,3}$. These two approaches are often used to estimate the amount of dark matter (DM) in the disk. It was argued that the stellar kinematics are inconsistent with a higher end estimate of the central mass density ${ }^{7}$, implying that DM in the disk may account for the deficient mass. Since the Hipparcos data set is presently the most extensive astrometric data available, analyses based on it should be considered the most reliable. They appear to converge towards lower values of 0.10 to $0.13 \mathrm{M}_{\odot} \mathrm{pc}^{-32,3}$, suggesting that the unobserved DM in the disk is at most the amount expected from a spherical or oblate DM halo, around 0.01 to $0.03 \mathrm{M}_{\odot} \mathrm{pc}^{-3}$. The DM density is important because its exact value is essential for searches of weakly interacting massive particles ${ }^{8}$.

The third approach to derive the density of the galactic disk is based on the periodicity of the solar systems vertical oscillation (VO). The periodic perturbation of the Oort cloud should increase the population of comets crossing the Earth pathway, potentially leaving an imprint in the terrestrial cratering record. Estimates for cratering periodicity range from 26 to $36 \mathrm{Ma}$ (e.g., ref. 9), but the sparse statistics are disputed ${ }^{10}$.

Paleo-climatic records could serve as alternative chronometers if a physical mechanism exists to link the solar system's vertical motion to the terrestrial climate. Several suggested mechanisms could potentially do so: 1) perturbation of comets in the Oort cloud that, after disintegration into dust, could potentially cool the climate ${ }^{11}$, 2) collision with interstellar gas clouds ${ }^{12}$, 3) climate modulation via cosmic rays ${ }^{13-15}$. In the latter case, the VO would translate into an oscillatory variation in the cosmic ray flux, because the cosmic rays density depends on the distance from the galactic plane ${ }^{16}$. In addition, the lower interstellar pressure would allow the heliosphere to puff up and increase the cosmic ray energy loses as they propagate into the solar system ${ }^{17}$.

Since the cosmic ray mechanism can potentially explain climate variations on longer time scales ${ }^{18}$, we will assume that the link does indeed operate and with it construct in what follows a model for the average climate. However, two points should be noted. First, any VO/climate correlation by itself does not prove that the climate link is through any particular mechanism. Second, the cosmic ray climate link is controversial and it should therefore not be taken for granted. For example, various criticisms were raised on the validity and implications of 


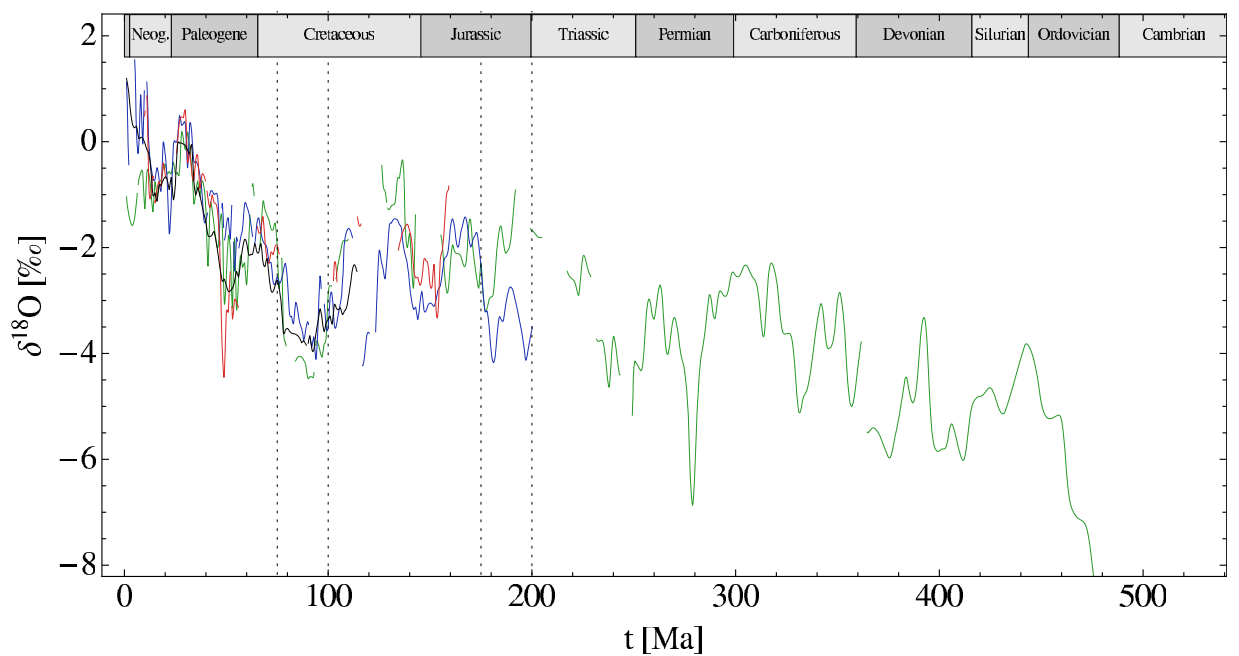

Figure $1 \mid$ The Gaussian filtered $\delta^{18} \mathrm{O}$ data, separated into four groups. The green line is the low-latitude, blue the mid-latitude, red the high-latitude, and the black line the deep sea subset. The latter three subsets were shifted to minimize the $\chi^{2}$ between them and the low-latitude subset (see Supplementary Materials for details). Note that the low-latitude data show a warming for the past 15 Ma while the three other subsets exhibit cooling. Note also the data gaps around 110 and $210 \mathrm{Ma}$. The dotted vertical lines denote time intervals used for splicing the different combinations of subsets.

the long time scale correlations ${ }^{19}$, the cloud cover cosmic-ray correlation on the decade time scale ${ }^{20}$ and during Forbush decreases ${ }^{21}$, or whether the observed link between atmospheric ionization and the nucleation of small particles can affect the number density of cloud condensation nuclei ${ }^{22}$.

Here we use a new database of $\delta^{18} \mathrm{O}$ values over $488 \mathrm{Ma}$, which covers almost the entire Phanerozoic, and that transcend the aforementioned limitations. We will show that it exhibits a very clear 32 Ma periodicity.

The data. The original database of some $4500 \delta^{18} \mathrm{O}$ measurements from low-Mg calcitic fossils ${ }^{23}$, a reflection of Phanerozoic climate ${ }^{24}$, was shown to encompass periodicities of about 145 and $32 \mathrm{Ma}^{25}$. This database was later updated ${ }^{26}$ and once more prior to the present study, while keeping its original structure. It now includes over $24000 \delta^{18} \mathrm{O}$ values. It lists separately data for the deep sea $(>300 \mathrm{~m}$ depth) and surficial waters, the latter separately for the low-, mid- and high-latitudes and, where possible, also for the subtropical realms. Due to biological evolution, and the resulting diversity of fossil phyla, none of the subsets covers the entire $542 \mathrm{Ma}$ of the Phanerozoic time span. This necessitates splicing of the partial records. The entire $\delta^{18} \mathrm{O}$ and $\delta^{13} \mathrm{C}$ database and its reduction procedures are described in the Methods below, and elaborated in the Supplementary Materials.

The secular $\delta^{18} \mathrm{O}$ trend homogenized into $1 \mathrm{Ma}$ bins is reproduced in Fig. 1. Visual inspection of this figure, spectral (Supplementary Fig. S3) and wavelet analyses (Supplementary Figs. S4, S5) show that all subsets (tropical to high-latitude realms) exhibit a similar 30 to $40 \mathrm{Ma}$ oscillation, confirming its robustness and global validity. This permits the splicing procedure over the almost entire Phanerozoic, providing corrections for offsets relative to the low-latitude subset, the latter covering most of the studied time span, are taken into account. We tested 5 possible splicing combinations and they all yielded similar outcomes (see Supplementary Fig. S5), with only small quantitative differences. All subsequent discussion is therefore based on a "master" set that splices the two most complete subsets, the mid-latitude one for the $<200 \mathrm{Ma}$ interval and the low-latitude one for the time interval $>200 \mathrm{Ma}$. The detrended and high pass filtered $\delta^{18} \mathrm{O}$ data of this "master" (ML200) set are reproduced in fig. 2.

The model. To test whether the apparent $\delta^{18} \mathrm{O}$ oscillations are related to the galactic VO of the solar system, we need to construct a model and compare it with the above data. Such a model requires two components. One is integrating the galactic orbit, and in particular, the VO, while the second component is linking the distance from the galactic place to the terrestrial climate.

For the orbit integration, we assume that there is a negligible drop of the density with distance from the galactic plane, implying that the vertical oscillation is harmonic. A realistic density fall will typically introduce a $10 \%$ error $^{2}$. Namely, the density fitted describes an average density experienced by the solar system, not the actual density at the galactic plane. We also assume that the density falls exponentially with radial distance, such that it can be written as:

$$
\rho(r, z)=\rho_{0} \exp (-r / R) .
$$

For an $R=3.5 \pm 0.5 \mathrm{kpc}$ decay length ${ }^{1}$, a $650 \mathrm{pc}$ radial epicyclic motion of the solar system (RO) would give rise to a $17 \pm 3 \%$ variation in the density (See $\S S 7$ for a summary of the kinematic parameters). This variation should be higher if any flaring of the disk is present. For comparison, the second largest modulation, due to the spiral arms, is expected to result in only a 5 to $10 \%$ density variation $^{27,28}$.

The orbit can then be integrated to give the $\mathrm{VO}$ and a $\mathrm{RO}$, as described in Supplementary Materials S3. One of the more interesting aspects of this orbit is the coupling between the average disk density and the vertical oscillation. When the solar system is closer to the galactic centre, the density is higher and the vertical oscillation is both faster and with a smaller amplitude than when it is further out.

The main hypothesis in the model is that the average global temperature depends on the distance from the galactic plane. The lowest order expansion of any symmetric relation should be second order, that is,

$$
\delta^{18} \mathrm{O}=A_{18}+B_{18} z^{2} .
$$

The constants will depend on the actual physical link between $z$ and the terrestrial temperature, if such a link exists. Again we note that we do not assume any particular model to be responsible for this link, however, we assume for practical reasons that it is the cosmic ray climate link ${ }^{13}$ since it provides us with quantitative predictions for the actual temperature variations expected.

We expect the cosmic rays to diffuse out of the galactic plane, giving a drop with height. Since the cosmic ray halo is much larger than the typical $100 \mathrm{pc}$ amplitude of the $\mathrm{VO}^{29}$, the lowest order expansion should be adequate. Because the oscillations are nearly 


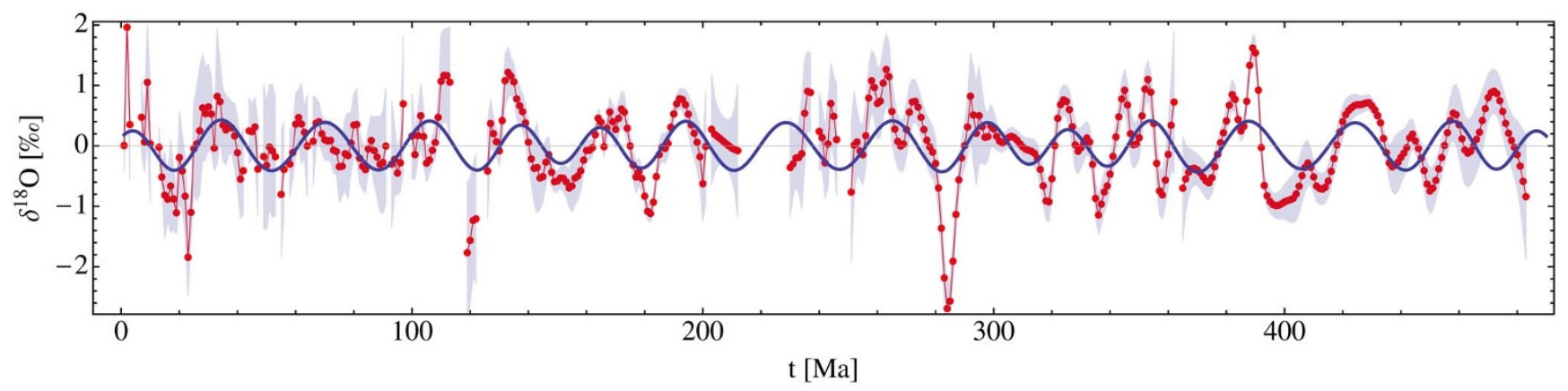

Figure $2 \mid$ The linearly detrended and high pass filtered ML200 $\delta^{18} \mathrm{O}$ dataset (in red) for Fourier modes shorter than 49 Ma. This is because we do not model processes on longer time scales. The simulated VO motion of the solar system in the galaxy (blue) has a secondary frequency modulation caused by the epicyclic motion of the solar system that generates slightly shorter VO periods around $130 \mathrm{Ma}$ and $300 \mathrm{Ma}$ and longer ones in between.

Because the vertical potential changes adiabatically with the epicyclic motion, the vertical amplitude is larger when the period is longer. The shaded region denotes the $95 \%$ confidence range for the measured $\delta^{18} \mathrm{O}$ obtained from the finite number of data points in each bin and the variance in the data.

harmonic, the $T(z)$ relation associated with eq. 2 would then be close to sinusoidal, with an amplitude and frequency that vary with the RO oscillations.

As an example, if the cosmic ray density at the maximum amplitude $z_{\text {max }}$ is $30 \%$ smaller than at the plane, it would translate into a 2 $\pm 1^{\circ} \mathrm{C}$ temperature variation ${ }^{30}$ (with the plane being colder). This would correspond to $B_{18} / z_{\text {max }}^{2} \approx 0.5 \pm 0.25 \%$ without ice-volume effects, or more with.

The free parameters include the present vertical location $z$ and velocity $v_{z, 0}$, the average density $\rho_{0}$ and its radial gradient $\rho_{0} / R$, the amplitude of the radial oscillation $a_{R}$ and time of perigalacticon $t_{p}$, as well as the parameters $A_{18}$ and $B_{18}$ appearing in eq. 2 . Since many of the parameters have independent observations, the best fit parameters in any viable model should be consistent with the observations. Note also that there is a degeneracy between $z_{\text {max }}^{2}$ and $B_{18}$, as well as between the radial density gradient and the amplitude of the radial motion.

\section{Results}

We optimize these free model parameters by minimizing the residuals between the predicted model and the detrended "master" set (fig. 2). Because the data is noisy, with many local minima, we employ a genetic algorithm for minimization. The best fit gives an average VO of nearly $32 \mathrm{Ma}$, with a $175 \mathrm{Ma}$ frequency modulation by RO. We use the bootstrap method on the different spliced datasets to estimate the statistical and systematic errors, as described in §S4 of the Supplementary Materials. For the exact fit parameters see Table 1.

The plot of $\delta^{18} \mathrm{O}$ as a function of the modeled vertical height $z$ in the galactic disk (fig. 3) demonstrates that the average $\delta^{18} \mathrm{O}(z)$ can be adequately described with a quadratic form in $z$ that is assumed in the model fit. The figure also shows that these $\delta^{18} \mathrm{O}$ oscillations are present in all latitudinal subsets, again confirming the robustness and global validity of the observations. The total $\delta^{18} \mathrm{O}$ variation is about $1 \%$, roughly $4{ }^{\circ} \mathrm{C}$, or less if the dynamics of ice caps accounts for some of the oscillations in the isotope signal. The impact of the secondary modulation of $\mathrm{VO}$ by $\mathrm{RO}$, which explains the 30-40 Ma range of values around the primary $32 \mathrm{Ma}$ frequency in the wavelet analysis (Supplementary Figs. S3-S5), is illustrated in fig. 4.

Statistical significance of the $32 \mathrm{Ma}$ signal. In order to assess the statistical significance of the $32 \mathrm{Ma}$ signal, we adopt the Multitaper Method (MTM) often used when studying climate signals. The method and the specific toolkit used are described at length in ref. 31. It allows us to estimate the amount of structure at different spectral bands and to assess the statistical significance by estimating the amount of noise level as a function of frequency expected for the null hypothesis.

Fig. 5 describes the MTM spectrum. Because there is no particular preference for any noise model, we conservatively assess the noise level as a function of frequency under the assumption of an AR(1) model $^{31}$. This modeling allows for more noise to occur naturally at low frequencies, ensuring that the significance is not artificially high.

The spectrum reveals two statistically significant peaks at low frequencies, around $150 \mathrm{Ma}$ and around $30 \mathrm{Ma}$, with a significance that is more than $99.9 \%$ and $99.99 \%$ respectively. If we assume a "locally white" model for the null hypothesis instead of an $\operatorname{AR}(1)$ process, the significance is $99.99 \%$ per frequency bin for both peaks. (The significance for pure "white noise" will be much higher).

Since we expect the galactic half period to be 30 to $42 \mathrm{Ma}^{3,4}$, and since the frequency resolution is about $0.001 \mathrm{Ma}^{-1}$, there are roughly 10 frequency bins in which the galactic signal can appear. Thus, the probability that the given frequency band will randomly yield a signal which is as large as that observed in any of the bins, is about $10^{-3}$.

Table 1 reveals that the nominal phases of the geological $32 \mathrm{Ma}$ signal and the astronomical data are consistent within $1.1 \mathrm{Ma}$ of each other (with typical errors of 1.2 to $1.5 \mathrm{Ma}$ on each measurement). Thus, there is a about a $15 \%$ probability that the two unrelated signals will have a phase difference which is as small as the one observed or within the combined error. This implies that the probability that there will be a random fluctuation giving a galactic like oscillation (i.e., within 30 to $42 \mathrm{Ma}$ period) and a phase which is consistent with the astronomical data is about $1.5 \times 10^{-4}$.

The probability that this random fluctuation would also have a secondary modulation that is consistent both in phase and period with the radial epicyclic motion is smaller by at least an order of magnitude. Thus the statistical significance of the $32 \mathrm{Ma}$ signal and its secondary modulation is of order $\mathcal{O}\left(10^{-5}\right)$.

\section{Discussion}

Given the consistency between the vertical and radial oscillations and the paleoclimate data, and the low probability that it could be mimicked by random fluctuations, we conclude with high confidence that the terrestrial temperature has a component which is quadratic in the distance from the galactic plane. Although this can be naturally explained through the cosmic ray climate $\operatorname{link}^{13}$, the observations by themselves do not prove it.

In addition, it should be noted that although a galactic driver can naturally explain a stable $\sim 32 \mathrm{Ma}$ cycle, there are terrestrial processes that could drive climate variations on the $\sim 32$ Ma time scale as well. The most prominent is probably mantle convection periodically producing plumes that result in large volcanic eruptions/igneous provinces. These eruptions will in turn add aerosols and carbon 
Table 1 | Summary of the paleoclimate derived galactic parameters

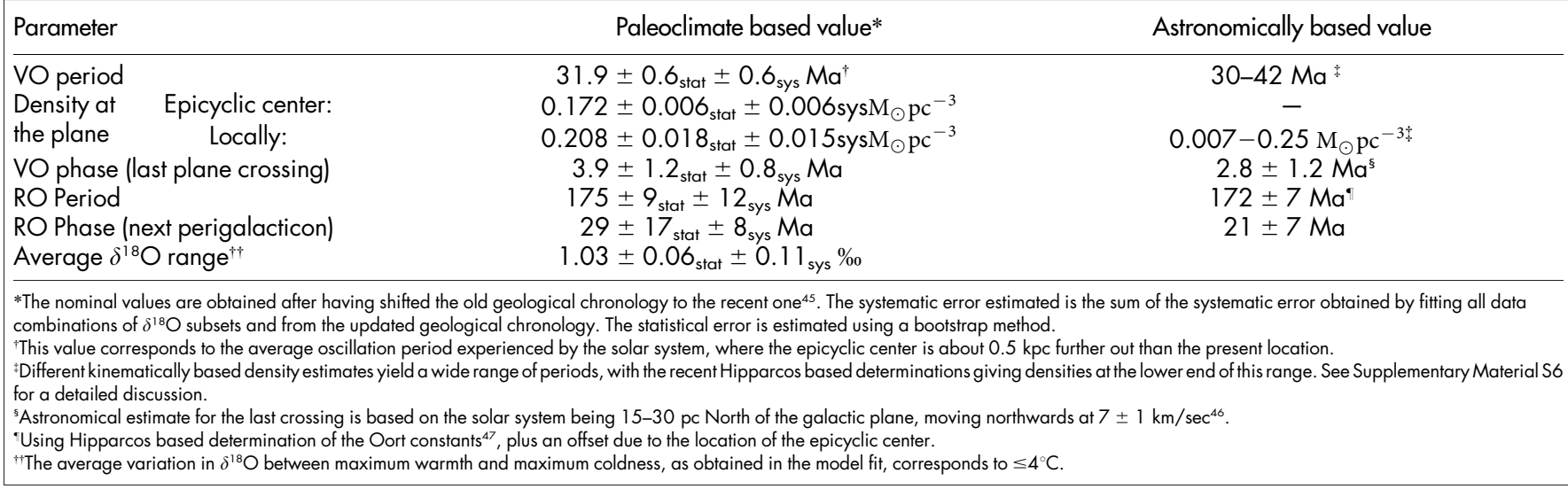

dioxide to the oceanic-atmospheric system ${ }^{32}$ and either cool or warm the climate. The influence of the Earth mantle connection could also drive changes or even reversals in Earth's magnetic field ${ }^{33,34}$, which would modulate the atmospheric ionization. The advantage of the proposed galactic forcing over a terrestrial driver is that it will produce a gradual (sinusoidal) fluctuations as found for most of the $\delta^{18} \mathrm{O}$ record with a relatively steady periodicity, while volcanic forcing will more likely produce random abrupt perturbations followed by gradual relaxations to climate base levels (e.g., "volcanic winters"35). The non-gradual behaviour would also characterize other galactic forcings, such as periodic asteroid/comet bombardments triggered by the galactic tides ${ }^{36}$.

As a side note, there is also evidence for a $\sim 60$ Ma periodicity in the literature ${ }^{37-42}$. For example, Meyers \& Peter $^{38}$ find a $56 \pm 3 \mathrm{Ma}$ period in the sedimentation record, which can be explained as an orogenic oscillator or as oscillatory dynamics in the mantle convection. Thus, these $\sim 60$ Ma periodicities are probably unrelated to the $32 \mathrm{Ma}$ cycle discussed here, unless there is a very large north-south asymmetry relative to the galactic plane, and the 60 Ma cycle can be shown to be in phase with double the $32 \mathrm{Ma}$ cycle discussed here.

The high accuracy of the estimated VO periodicity also enables estimating the density at the galactic plane, and may potentially point to an interesting conundrum. Our effective density (for an equivalent homogeneous disk) is $0.172 \pm 0.006_{s t a t} \pm 0.006_{s y s} \mathrm{M}_{\odot} \mathrm{pc}^{3}$ at the radial center of the epicyclic motion, and $0.208 \pm 0.018_{\text {state }} \pm$ $0.015_{\text {sys }} \mathrm{M}_{\odot} \mathrm{pc}^{-3}$ at the present galactic radius (near perigalacticon, see Table 1). These values are inconsistent with the density of $0.103 \pm 0.010 \mathrm{M}_{\odot} \mathrm{pc}^{-3}$ obtained by analyzing the dynamics of A \& $\mathrm{F}$ stars in the Hipparcos catalogue ${ }^{4}$. Some of this inconsistency can be explained given that the different numbers do not reflect the same density. For example, the different model parameters may have changed over the past $500 \mathrm{Ma}$ due to diffusion in parameter space, such that the $32 \mathrm{Ma}$ reflects the average witnessed by the solar system over the past $500 \mathrm{Ma}^{43,44}$, and not the exact parameters today. In addition, we show in the Supplementary Materials that the Hipparcos based determination has a large unaccounted systematic uncertainty because the local stellar population is not dynamically relaxed. It is large enough to explain the whole inconsistency. It is worth to note in this respect that the GAIA star catalogue will be published in the near future. Since it will be much more extensive than the Hipparcos catalogue, both in terms of number of stars and their distance from the solar system, we expect under the above model to see convergence between the astronomically derived quantities and their respective geological counterparts. On the other hand, a more extensive geological database could provide additional evidence in the form of a second "frequency modulation" that corresponds to the density variations associated with the spiral arm passages. These variations are 2-3 times smaller than the $\mathrm{RO}$ and therefore harder to detect.

The density at the galactic plane should also be compared to the observed baryonic matter and the estimated halo dark matter densities, which only contribute $0.09-0.10 \mathrm{M}_{\odot} \mathrm{pc}^{-3}$ and $0.01-0.03$ $\mathrm{M}_{\odot} \mathrm{pc}^{-3}$ respectively (see ref. 4 and references therein). This discrepancy may imply the presence of a large unaccounted component that is not the usual halo dark matter.

\section{Methods}

Raw data. The first step in the present work was to expand the previously available database of $\delta^{18} \mathrm{O}$ and $\delta^{13} \mathrm{C}$ measurements for low-magnesium calcite shells of marine

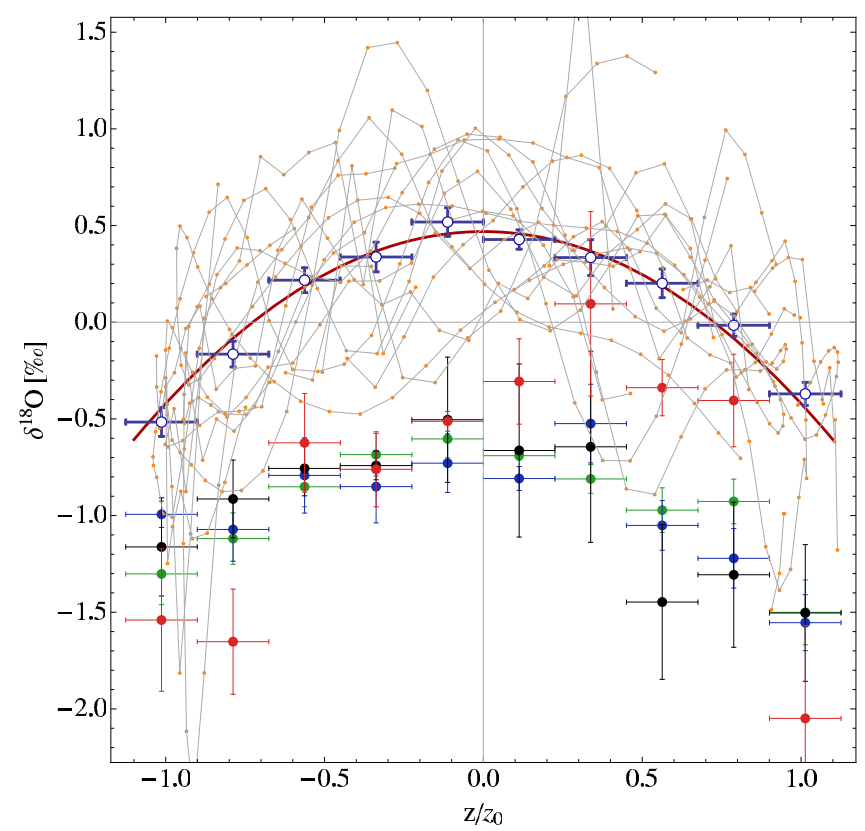

Figure $3 \mid$ The "master" set $\delta^{18} \mathrm{O}$ data (Fig. 2) plotted against the modeled vertical location in the galaxy. Normalization is relative to the maximal $z_{0}$ of the recent maximal excursion around $20 \mathrm{Ma}$ ago. The small orange dots are the actual $1 \mathrm{Ma}$ binned data. The thick blue error bars are the averages of the combined data binned to 10 equal vertical bins. The red curve is a parabolic best fit: $\delta^{18} \mathrm{O} /[\% \mathrm{o}]=0.42-0.09\left(z / z_{0}\right)-0.76\left(z / z_{0}\right)^{2}$. The additional points are a coarser binning of the latitudinally separated subsets (colours are the same as in Fig. 1). Because of the poor coverage, the latitudinal data subsets cannot be high pass filtered. Instead, they have the low pass component of the "master" set removed. For legibility, an offset of $-1 \%$ is applied. The fact that a similar vertical dependence appears in all 4 unrelated subsets indicates that the phenomenon is a global one. 


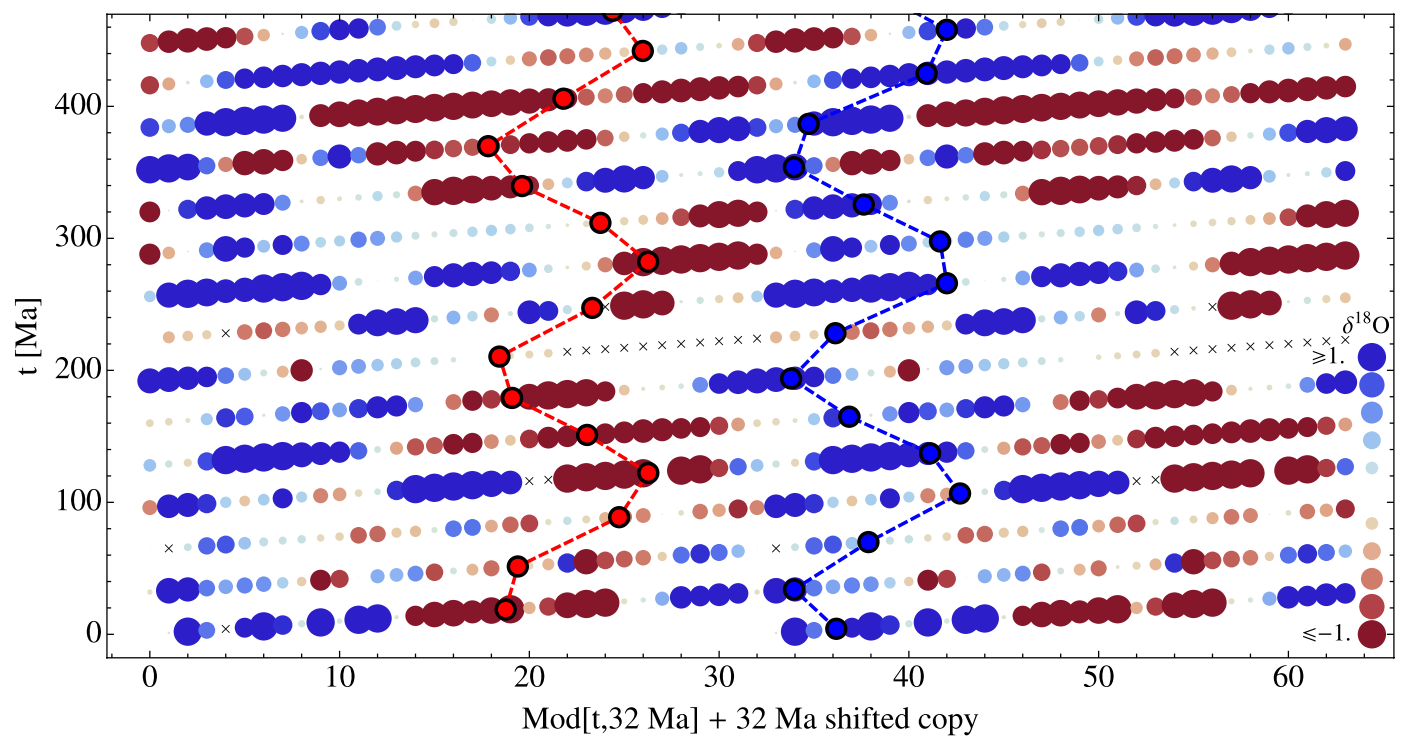

Figure $4 \mid$ A raster plot of the detrended 1 Ma binned master set of $\boldsymbol{\delta}^{18} \mathrm{O}$ data. The vertical axis spans the Phanerozoic. The horizontal axis is the time folded over a 32 Ma period. For convenience, the horizontal axis is duplicated and shifted by $32 \mathrm{Ma}$. The blue and red circles (connected by dashed lines) are the modeled galactic plane crossings (blue) and the maximal excursions from the plane (red), respectively. An unmodulated 32 Ma signal would appear as a vertical line over the entire $490 \mathrm{Ma}$ interval. The apparent modulation due to the radial epicyclic motion (RO) of the solar system is expressed as sinusoidal variations of this line. Xs denote bins with insufficient measurements. The disk radii and colour correspond to the detrended and high pass filtered $\delta^{18} \mathrm{O}$ signal, as given by the scale on the right (in \%).

fossils for the Phanerozoic (last $542 \mathrm{Ma}$ ). This present database is expanded by almost $30 \%$ relative to the previous compilation ${ }^{26}$, to about 24,000 measurements (Table S1), with brachiopods, foraminifera and belemnites accounting for the bulk of the samples. It includes data from more than 20 new references that were published prior to July 2011 (see the 2nd sheet of Datafile S1). More information about the compilation is available in the Supplementary Materials. The database itself can be downloaded as Data File S1.

Homogenized time series. The first step in the data reduction process was to construct temporally homogeneous time series at equidistant $1 \mathrm{Ma}$ intervals. This was carried out by Gaussian filtering the raw data of each habitat (tropical to highlatitude) separately. The second step was to derive the average $\delta^{18} \mathrm{O}$ offsets between the low altitude and each of the other habitats, by comparing the average $\delta^{18} \mathrm{O}$ over periods with sufficient data in each habitat pair. Because no habitat has a complete coverage of the last $490 \mathrm{Ma}$, the third step was to combine the data sets by splicing different habitats together, once the aforementioned offsets are applied to the time series. This step produced 5 different sets spanning the last $490 \mathrm{Ma}$. The different sets were all used in the analysis, with the variations giving a handle on possible systematic errors. The nominal set was the ML200 which includes the midlatitude data over the last $200 \mathrm{Ma}$, and the low-altitude data before that. It includes only one splice and

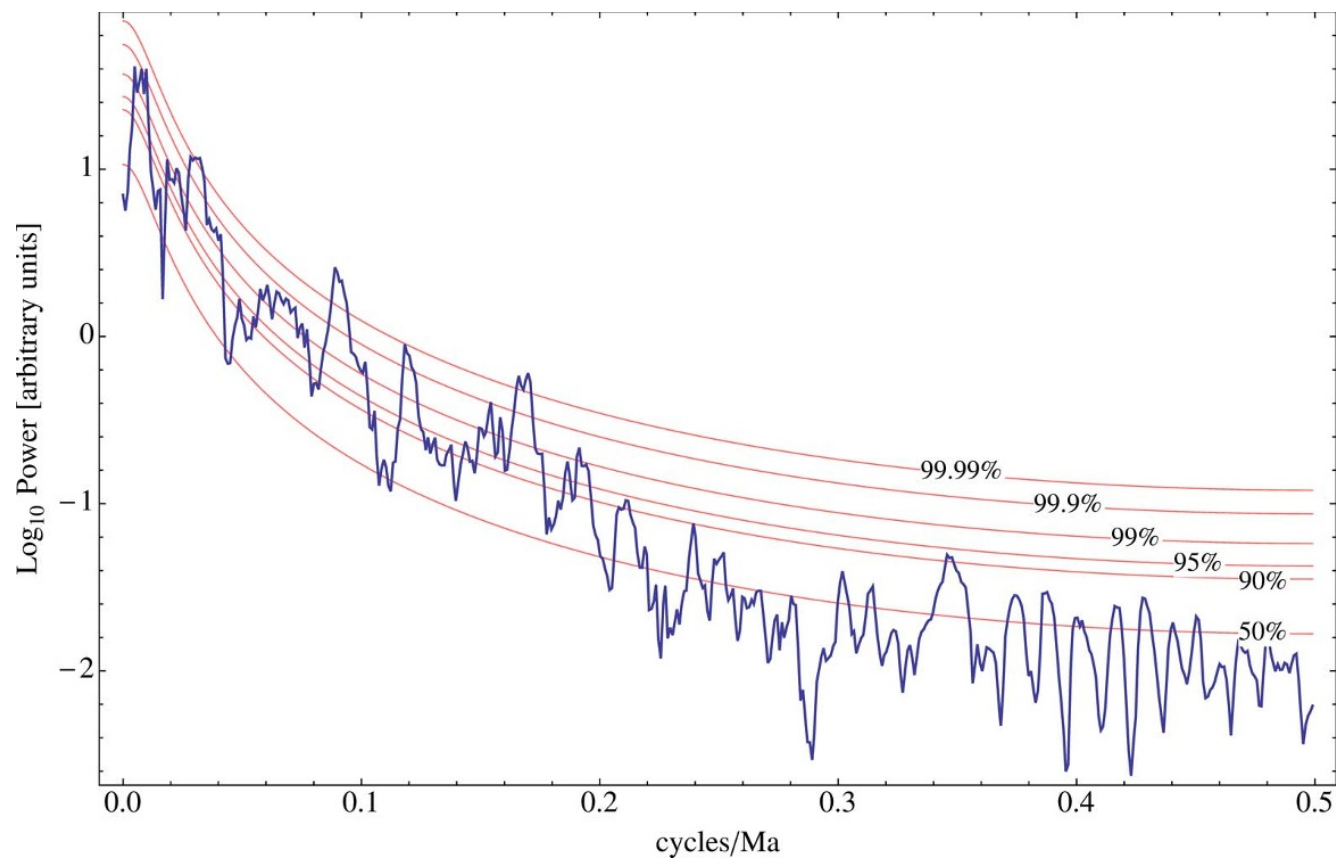

Figure $5 \mid$ The Multitaper Spectrum of the DML75 dataset, using 3 tapers. Without a good model for the noise estimate, the noise level is conservatively estimated assuming an AR(1) model, while allowing for more noise at low frequencies than a "white" noise model would. See ref. 31 for a description of the method. The dominant low frequency peaks are at $f \sim 0.006$ cycle/Ma and $f \sim 0.03$ cycle/Ma which are also clearly evident in the wavelet and Fourier analyses (see §S2.5 of the supplementary material). Significant higher frequencies are also present. However, they are absent from the wavelet and Fourier analyses. It is therefore not clear whether they correspond to real climate variations or to artifacts in the dataset. 
avoids the recent low-latitude data that show atypical low $\delta^{18} \mathrm{O}$ values for the last $15 \mathrm{Ma}$, if compared to all the habitats. Although the raw data is based on the 2004 geological chronology, the time scale was adjusted to the new 2012 chronology $^{45}$.

1. Binney, J. \& Tremaine, S. Galactic dynamics (Princeton University Press, 1987).

2. Bahcall, J. N. \& Bahcall, S. The sun's motion perpendicular to the galactic plane. Nature 316, 706-708 (1985).

3. Stothers, R. B. Galactic disc dark matter, terrestrial impact cratering and the law of large numbers. Mon. Not. Roy. Astro. Soc. 300, 1098-1104 (1998).

4. Holmberg, J. \& Flynn, C. The local density of matter mapped by Hipparcos. Mon. Not. Roy. Astro. Soc. 313, 209-216 (2000).

5. Garbari, S., Read, J. I. \& Lake, G. Limits on the local dark matter density. Mon. Not. Roy. Astro. Soc. 416, 2318-2340 (2011).

6. Oort, J. H. Note on the determination of $K_{z}$ and on the mass density near the Sun. Bull. Astron. Inst. Netherlands 15, 45 (1960).

7. Bahcall, J. N., Flynn, C. \& Gould, A. Local dark matter from a carefully selected sample. Astrophys. J. 389, 234-250 (1992).

8. Gaitskell, R. J. Direct detection of dark matter. Ann. Rev. Nucl. Part. Sci. 54, 315-359 (2004).

9. Napier, W. M. Evidence for cometary bombardment episodes. MNRAS 366, 977-982 (2006).

10. Lyytinen, J., Jetsu, L., Kajatkari, P. \& Porceddu, S. Detection of real periodicity in the terrestrial impact crater record: quantity and quality requirements. $A \& A 499$, 601-613 (2009).

11. Hoyle, F. \& Wickramasinghe, C. Comets, ice ages, and ecological catastrophes. Astrophys. Sp. Sci. 53, 523-526 (1978).

12. Yabushita, S. \& Allen, A. J. On the effect of interstellar matter on terrestrial climate. The Obs. 105, 198-200 (1985).

13. Svensmark, H. Influence of Cosmic Rays on Earth's Climate. Phys. Rev. Lett. 81, 5027-5030 (1998).

14. Svensmark, H., Bondo, T. \& Svensmark, J. Cosmic ray decreases affect atmospheric aerosols and clouds. Geophys. Res. Lett. 36, 15101 (2009).

15. Svensmark, H., Enghoff, M. B. \& Pedersen, J. O. P. Response of cloud condensation nuclei $(>50 \mathrm{~nm})$ to changes in ion-nucleation. Phys. Lett. A 377 2343-2347 (2013)

16. Beuermann, K., Kanbach, G. \& Berkhuijsen, E. M. Radio structure of the GalaxyThick disk and thin disk at $408 \mathrm{MHz}$. AઐA 153, 17-34 (1985).

17. Shaviv, N. J. in Solar Journey: The Significance of our Galactic Environment for the Heliosphere and Earth (ed. Frisch, P. C.) 99-132 (Astrophysics and Space Science Library, Vol. 338, Springer, 2006)

18. Shaviv, N. J. \& Veizer, J. Celestial driver of Phanerozoic climate? GSA Today 13 $4-10$ (2003).

19. Royer, D. L., Berner, R. A., Montañez, I. P., Tabor, N. J. \& Beerling, D. J. $\mathrm{CO}_{2}$ as a primary driver of Phanerozoic climate. GSA Today 14, 4-10 (2004).

20. Kristjánsson, J. E. et al. Cosmic rays, cloud condensation nuclei and clouds-a reassessment using MODIS data. Atmos. Chem. Phys. 8, 7373-7387 (2008).

21. Laken, B., Kniveton, D. \& Wolfendale, A. Forbush decreases, solar irradiance variations, and anomalous cloud changes. J. Geophys. Res. (Atmos.) 116, 9201 (2011).

22. Pierce, J. R. \& Adams, P. J. Can cosmic rays affect cloud condensation nuclei by altering new particle formation rates? Geophys. Res. Lett. 36, 9820 (2009).

23. Veizer, J. et al. ${ }^{87} \mathrm{Sr} /{ }^{86} \mathrm{Sr}, \delta^{13} \mathrm{C}$ and $\delta^{18} \mathrm{O}$ evolution of Phanerozoic seawater. Chem. Geol. 161, 59-88 (1999).

24. Veizer, J., Godderis, Y. \& Francois, L. M. Evidence for decoupling of atmospheric $\mathrm{CO}_{2}$ and global climate during the Phanerozoic eon. Nature 408, 698-701 (2000).

25. Prokoph, A. \& Veizer, J. Trends, cycles and nonstationarities in isotope signals of Phanerozoic seawater. Chem. Geol. 161, 225-240 (1999).

26. Prokoph, A., Shields, G. A. \& Veizer, J. Compilation and time-series analysis of a marine carbonate $\delta^{18} \mathrm{O}, \delta^{13} \mathrm{C},{ }^{87} \mathrm{Sr} /{ }^{86} \mathrm{Sr}$ and $\delta^{34} \mathrm{~S}$ database through Earth history. Earth-Sci. Rev. 87, 113-133 (2008).

27. Roberts, W. W. Large-scale shock formation in spiral galaxies and its implications on star formation. Astrophys. J. 158, 123 (1969).

28. Roberts Jr, W. W. Application of the Density-Wave Theory of Spiral Structure: Shock Formation Along the Perseus Arm. Astrophys. J. 173, 259 (1972).
29. Strong, A. W., Moskalenko, I. V. \& Ptuskin, V. S. Cosmic-Ray Propagation and Interactions in the Galaxy. Ann. Rev. Nuc. Part. Sci. 57, 285-327 (2007).

30. Shaviv, N. J. On climate response to changes in the cosmic ray flux and radiative budget. J. Geophys. Res. 110, A08105 (2005).

31. Ghil, M. et al. Advanced spectral methods for climatic time series. Rev. Geophys. 40, 3-1 (2002)

32. Caldeira, K. \& Rampino, M. R. The Mid-Cretaceous Super Plume, carbon dioxide, and global warming. Geophys. Res. Lett. 18, 987-990 (1991).

33. Larson, R. L. \& Olson, P. Mantle plumes control magnetic reversal frequency. Earth Plan. Sc. Lett. 107, 437-447 (1991).

34. Courtillot, V. \& Olson, P. Mantle plumes link magnetic superchrons to phanerozoic mass depletion events. Earth Plan. Sc. Lett. 260, 495-504 (2007).

35. Rampino, M. R. \& Self, S. Climate-volcanism feedback and the Toba eruption of 74,000 years ago. Quater. Res. 40, 269-280 (1993).

36. Rampino, M. R. Role of the galaxy in periodic impacts and mass extinctions on the Earth. Geo. Soc. Am. Sp. Pap. 356, 667-678 (2002).

37. Rohde, R. A. \& Muller, R. A. Cycles in fossil diversity. Nature 434, 208-210 (2005).

38. Meyers, S. R. \& Peters, S. E. A 56 million year rhythm in North American sedimentation during the Phanerozoic. Earth. Plan. Sc. Lett. 303, 174-180 (2011).

39. Melott, A. L., Bambach, R. K., Petersen, K. D. \& McArthur, J. M. An 60-MillionYear Periodicity Is Common to Marine $87 \mathrm{Sr} / 86 \mathrm{Sr}$, Fossil Biodiversity, and LargeScale Sedimentation: What Does the Periodicity Reflect? J. Geo. 120, 217-226 (2012).

40. Prokoph, A., Ernst, R. E. \& Buchan, K. L. Time-series analysis of large igneous provinces: 3500 Ma to present. J. Geo. 112, 1-22 (2004)

41. Prokoph, A., Rampino, M. R. \& El Bilali, H. Periodic components in the diversity of calcareous plankton and geological events over the past 230 Myr. Palaeogeo. Palaeoclim. Palaeoeco. 207, 105-125 (2004).

42. Prokoph, A., El Bilali, H. \& Ernst, R. Periodicities in the emplacement of large igneous provinces through the Phanerozoic: relations to ocean chemistry and marine biodiversity evolution. Geosc. Front. 4, 263-276 (2013).

43. Wielen, R. The diffusion of stellar orbits derived from the observed agedependence of the velocity dispersion. A\&A 60, 263-275 (1977).

44. Shaviv, N. J. The spiral structure of the Milky Way, cosmic rays, and ice age epochs on Earth. N. Astron. 8, 39-77 (2003).

45. Gradstein, F. M., Ogg, G. \& Schmitz, M. The Geologic Time Scale 2012 (Elsevier, 2012).

46. Joshi, Y. C. Displacement of the Sun from the Galactic plane. Mon. Not. Roy. Astro. Soc. 378, 768-776 (2007).

47. Feast, M. \& Whitelock, P. Galactic kinematics of Cepheids from HIPPARCOS proper motions. Mon. Not. Roy. Astro. Soc. 291, 683-693 (1997).

\section{Author contributions}

The geochemical data analyses and compilation was carried out by A.P. and J.V. The astrophysical modeling was done by N.J.S. The manuscript was written jointly by N.J.S., A.P. and J.V.

\section{Additional information}

Supplementary information accompanies this paper at http://www.nature.com/ scientificreports

Competing financial interests: The authors declare no competing financial interests.

How to cite this article: Shaviv, N.J., Prokoph, A. \& Veizer, J. Is the Solar System's Galactic Motion Imprinted in the Phanerozoic Climate? Sci. Rep. 4, 6150; DOI:10.1038/srep06150 (2014)

This work is licensed under a Creative Commons Attribution-NonCommercialNoDerivs 4.0 International License. The images or other third party material in this article are included in the article's Creative Commons license, unless indicated otherwise in the credit line; if the material is not included under the Creative Commons license, users will need to obtain permission from the license holder in order to reproduce the material. To view a copy of this license, visit http:// creativecommons.org/licenses/by-nc-nd/4.0/ 